\title{
Identificação de dor após orquiectomia em garrotes: desenvolvimento de escala análogo visual em comparação a parâmetros fisiológicos, padrões comportamentais e expressão facial $^{1}$
}

\author{
Heloisa G. Bertagnon ${ }^{2,3 *}$, Camila F. Batista², Jessyca B. Bellinazzi², \\ Mariana M. Coneglian ${ }^{3}$, Adriano F. Mendes ${ }^{3}$ e Alice M.M.P. Della Libera ${ }^{2}$
}

\begin{abstract}
Bertagnon H.G., Batista C.F., Bellinazzi J.B., Coneglian M.M., Mendes A.F. \& Della Libera A.M.M.P. 2018. [Pain identification after orchiectomy in young bulls: development of the visual analogue scale compared with physiological parameters, behavioral patterns and facial expression.] Identificação de dor após orquiectomia em garrotes: desenvolvimento de escala análogo visual em comparação a parâmetros fisiológicos, padrões comportamentais e expressão facial. Pesquisa Veterinária Brasileira 38(3):436-443. Departamento de Clínica Médica, Faculdade de Medicina Veterinária e Zootecnia, Universidade de São Paulo, Avenida Prof. Dr. Orlando Marques de Paiva 87, Cidade Universitária, São Paulo, SP 05508-270, Brazil. E-mail: hbertagnon@unicentro.br

The identification of pain in cattle and your relief, are essential for animal welfare, however there is still no ideal test for this evaluation. Some researchers have used serum cortisol, heart and respiratory rates for this assessment, while others use scales based on behavior or facial expressions. However, doesn't exist but a scale that takes into account the union of these identifiers for cattle. Furthermore, most researchers manipulate the animals to identify pain, which could mask the result. In this way, this paper proposed design and validate a visual analog scale for pain identification in cattle undergoing orchiectomy. For this, 8 Holstein calves with 200 days old and $250 \mathrm{~kg}$ live weight were submitted to orchiectomy with local anesthesia and analgesics. The identification of pain was based on physiological analysis (serum cortisol and respiratory rate), behavioral, and facial expression in the visual-analogue -30 (30 minutes before surgery) and 1, 3, 6, 12, 24, 72 and 420 hours after the procedure; and with the exception of cortisol, all analyzes were performed without the interference of the appraiser with the animal, by videos. It was noted that all the methods proposed were able to identify post orchiectomy pain in cattle, however the degree of pain were higher in different post-surgical times. There was poor correlation between the proposed models, because it was found limitations for most assessed methodologies. We concluded an association between various parameters of pain, as visual analogue scale, can increase the accuracy to identify pain orchiectomy in bulls

INDEX TERMS: Pain, orchiectomy, bulls, visual analogue scale, physiological parameters, behavioral patterns, facial expression, cattle, castration, cortisol, surgery.
\end{abstract}

\footnotetext{
${ }^{1}$ Recebido em 16 de setembro de 2016.

Aceito para publicação em 21 de março de 2017.

${ }^{2}$ Departamento de Clínica Médica, Faculdade de Medicina Veterinária e Zootecnia, Universidade de São Paulo (USP), Avenida Prof. Dr. Orlando Marques de Paiva 87, Cidade Universitária, São Paulo, SP 05508-270, Brasil.

${ }^{3}$ Departamento de Medicina Veterinária, Universidade Estadual do Centro-Oeste (Unicentro), Campus Cedeteg, Avenida Prof. Dr. Simeão Camargo Varella de Sá 3, Guarapuava, PR 85040-080, Brasil. *Autor para correspondência: hbertagnon@unicentro.br
}

RESUMO.- A identificação de dor em bovinos e seu alivio, são essenciais para o bem estar animal, todavia ainda não há um teste ideal para esta avaliação. Alguns pesquisadores têm utilizados cortisol sérico e frequências cardíaca e respiratória para esta avaliação, enquanto outros utilizam escalas baseadas em comportamento ou expressões faciais, não existindo uma escala que leve em consideração a união destes identificadores para bovinos. Além disso, a maioria dos pesquisadores manipulam os animais para identificar a dor, o que poderia 
mascarar os resultados. Desta maneira o presente trabalho propôs elaborar e validar uma escala análogo visual para identificação de dor em bovinos submetidos à orquiectomia. Para tanto 8 bovinos holandeses de 200 dias de vida e $250 \mathrm{~kg}$ de peso vivo foram submetidos a orquiectomia com previa anestesia local e uso de analgésicos. A identificação de dor foi baseada em analises fisiológica (cortisol sérico e frequência respiratória), comportamental, de expressão facial e análogo visual nos momentos -30 (30 minutos antes do procedimento) e 1, 3, 612 , 24, 72 e 420 horas após o procedimento; e com a exceção do cortisol, todas as analises foram realizadas sem a interferência do avaliador com o animal, por meio de filmagens de vídeos. Notou-se que todas as metodologias propostas foram capazes de identificar dor pós orquiectomia em bovinos, no entanto a graduação de maior dor foi em momentos pós-cirúrgicos distintos conforme o parâmetro avaliado. Assim houve fraca correlação entre os modelos propostos, pois se encontrou limitações para a maioria das metodologias avaliadas, concluindo-se que a associação entre os vários parâmetros de dor, tal qual o utilizado na escala análogo visual, aumenta a acurácia em identificar a dor após orquiectomia em bovinos.

TERMOS DE INDEXAÇÃO: Dor, orquiectomia, garrotes, escala análogo visual, parâmetros fisiológicos, padrões comportamentais, expressão facial, bovinos, castração, cortisol, cirurgia.

\section{INTRODUÇÃO}

O mercado consumidor de carne bovina é cada vez mais exigente o que gera uma série de sistemas e técnicas de manejo animal, que tem como intuito principal aumentar o lucro imediato do produtor, ignorando por vezes vários aspectos relacionados ao bem-estar do animal, como dor e estresse (Costa \& Cromberg 1997).

A castração de bezerros machos, por exemplo, é rotineiramente usada com a justificativa de facilitar o manejo destes animais e melhorar a qualidade da carne, entretanto há indícios de que sua execução gera dor nos animais mesmo quando protocolos analgésicos são utilizados (Ting et al. 2003, Thüer et al. 2007).

Estes processos dolorosos acarretam alterações fisiológicas manifestadas por aumento sérico de aldosterona, cortisol e catecolaminas (Fantoni \& Mastrocinque 2002), que aumentam o consumo de substratos pelos tecidos, resultando em perda de peso, alteração da imunidade, predisposição de animais a doenças, atraso no crescimento e prejuízos reprodutivos principalmente (Molony et al. 1995, Bellinazzi et al. 2013). Deste modo faz-se necessário não só a busca por alternativas viáveis para atenuar esse problema sem consequências econômicas, como também o desenvolvimento de estratégias para a identificação precoce da dor. No entanto os modelos animais para aferição de dor acabam sendo subjetivos, especialmente nos ruminantes, que têm comportamento relativamente tranquilo e os sinais de estresse e dor podem não ser claros (Millman 2013).

0 reconhecimento da dor e seu alívio é crítico para o bem-estar animal, e apesar de alguns progressos nesta área, ainda não há um teste ouro para avaliação de dor em ruminantes (Marti et al. 2010, Millman 2013, Müller 2015). Experimentos de analgesia pós-castração costumam utilizar como indicadores de dor os parâmetros fisiológicos de cortisol sérico e frequências cardíaca e respiratória (Molony et al. 1995,
Fitzpatrick et al. 2006), no entanto estes parâmetros podem ser afetados pela simples manipulação do animal não refletindo necessariamente dor no pós cirúrgico (Becker et al. 2012).

Alguns pesquisadores desenvolveram protocolos baseados em comportamentos e postura do animal para evidenciar dor após castração, levando em conta vocalização, alteração de postura, e atividades de pisoteio, lambedura, hipofagia e perda de peso (Robertson et al. 1994, Ting et al. 2003, Thüer et al. 2007, Marti et al. 2010), todavia a natureza linfática do animal desqualifica a avaliação de alguns destes comportamentos como a vocalização (Lay et al. 1992). Além disso, a perda de peso avalia mais a dor crônica que a dor aguda (Ting et al. 2003).

As expressões faciais são indicadores sensíveis de dor em humanos. 0 relaxamento ou tensão dos músculos da face estabelecem unidade de ação facial especificas indicativas de emoção (Ekman et al. 1980). Este mesmo modelo já foi extrapolado para alguns animais, como macacos e ovelhas (Tate et al. 2006), cães (Bloom \& Friedman 2013) e cavalos (Dalla Costa et al. 2014). Recentemente Müller (2015) validou este modelo para avaliar a demonstração de dor em bovinos submetidos à marcação da face com ferro quente, porém não se sabe se este modelo pode ser utilizado para outros estímulos dolorosos, como a castração.

Existe ainda escalas análogo visuais, que elencam uma serie de comportamentos, posturas, parâmetros vitais e expressões faciais para graduar a dor no paciente (Chambers et al. 2003, Dalla Costa et al. 2014), porém não encontrou-se na literatura, uma escala elaborada para ruminantes.

Desta maneira o presente trabalho teve como objetivo elaborar e validar uma escala análogo visual para identificação de dor em bovinos submetidos à orquiectomia cirúrgica com protocolo analgésico.

\section{MATERIAL E MÉTODOS}

Este projeto foi aprovado pela Comissão de Ética no Uso de Animais da Faculdade de Medicina Veterinária e Zootecnia da Universidade de São Paulo (FMVZ-USP), protocolo 2395/2011 em 26 de outubro de 2011.

Foram utilizados oito bezerros machos, da raça Holandesa preto e branca, sadios provenientes de propriedades criatórias próximas a São Paulo. Estes animais foram aclimatados por um período de três meses antecedendo o experimento em baias da FMVZ-USP, São Paulo-SP, ficando dois animais por baia. Simultaneamente, os dois bezerros de cada baia foram castrados cirurgicamente, às $8 \mathrm{~h} 00$, quando completaram 200 ( \pm 5 dias) dias de vida e $250( \pm 50 \mathrm{~kg})$ de peso vivo. A orquiectomia foi feita com o animal em decúbito lateral direito, derrubado pelo método italiano em colchão. Após assepsia e anestesia local infiltrativa com lidocaína sem vasoconstritor a $2 \%$, o procedimento cirúrgico foi realizado utilizando-se a técnica aberta e com ligadura do funículo espermático de acordo com o preconizado por Anderson (2007). Os animais receberam duas doses de $3 \mathrm{mg} / \mathrm{kg}$ de cetoprofeno intravenoso, sendo a primeira no pré-cirúrgico e a outra 24 horas depois. Após o procedimento, os animais retornavam a suas baias, onde tinham livre acesso à água e feno.

Os modelos de identificação de dor foram baseados em analises fisiológicas, comportamentais, expressão facial e análogo visual nos momentos -30 (30 minutos antes do procedimento) e 1, 3, 6, $12,24,72$ e 420 horas após o procedimento, sempre precedendo a 
coleta de sangue. Nestes momentos os animais estavam nas baias experimentais ( $4 \times 3 \mathrm{~m}$, sem janelas) contendo cama de maravalha, água e feno de tifton (Cynodon spp.).

Nas análises fisiológicas foram mensurados cortisol sérico e frequência respiratória (FR) dos animais. A aferição da FR foi realizada primeiro, por observador disposto do lado de fora das baias por inspeção visual. 0 cortisol sérico foi mensurado em amostras de sangue colhido da jugular externa em tubos sem anticoagulante por meio de um cateter $14 \mathrm{G}$ inserido e fixado na jugular dos animais. Esta dosagem foi realizada em o soro sanguíneo previamente congelado, por meio da técnica de ELISA, usando o kit comercial (Enzo Life Science ${ }^{\circledR}$, USA).

As analises comportamentais (Comp), foram mensuradas por meio da somatória das frequências de comportamento e da porcentagem do tempo dispendido em posturas antiálgicas, descritas no Quadro 1 baseado no modelo proposto por Thüer et al. (2007) e Marti et al. (2010) com modificações. A somatória destes índices foi transformada em escore de 4 pontos. Para estas análises, os animais foram filmados durante 10 minutos nos momentos propostos com uma câmera digital (Cybershot 1080, Sony) posicionada fora da baia do animal e três observadores diferentes, em modelo cego em relação aos momentos, registraram o comportamento dos mesmos.

Nas análises de expressão facial (Face), imagens que permitiam claramente a visualização da cabeça e face de cada animal foram extraídas dos vídeos, sendo duas imagens por momento. Cada imagem foi tratada, permitindo visualizar apenas a cabeça do animal sem o corpo e fornecida para dois observadores cegos em relação ao momento analisado. Observou-se a presença ou não das cinco unidades de ação facial (UAF): Colocação das orelhas para trás, dilatação das narinas, elevação/contração da face medial das pálpebras, elevação/contração da face lateral das pálpebras e abertura da boca, de acordo com o sugerido por Müller (2015). Estas UAF foram transformadas em escore de 0 a 5 pontos, sendo atribuído 1 ponto a cada UAF apresentada.

E na escala análogo visual (EA), foram analisadas variações fisiológicas, comportamentais e expressões faciais conforme Quadro 2 e Figura 1, montada pelos autores deste trabalho. Cada item analisado foi graduado de 0 a 3, sendo 0 considerado ausente e 3 a manifestação mais intensa. Apenas a posição de auto auscultação foi classificada como ausente ou presente, pois não há graduação para esta manifestação. A somatória total da tabela foi transformada em escores de 0 a 4 . Estes escores foram registrados por três observadores cegos em relação ao momento, que analisaram os vídeos anteriormente mencionados.

Os três observadores selecionados eram familiarizados com os bovinos e já conheciam estes animais desde a fase de aclimatação.

Análise estatística. A análise estatística dos dados foi realizada utilizando o software estatístico GraphPad InStat (GraphPad Software, La Jolla, USA) Foi verificada a normalidade da distribuição dos resultados, utilizando-se teste de AndersonDarling. Para a avaliação das diferenças entre as médias dos resultados obtidos, foram realizados os testes de análise de variância ANOVA One-way (Unstacked) para amostras repetidas e, quando identificada diferença, o teste de Tukey (para dados com distribuição normal). Para avaliação dos escores e do cortisol sérico (para dados que não apresentaram distribuição normal) foi utilizado teste para amostras repetidas Mann-Whitney e, quando identificada diferença, o teste de Dunn. Correlação de Spearman foi utilizada para verificar a relação entre análises fisiológicas, comportamentais, expressão facial e análogo visual. Para todos os resultados, foram consideradas significantes as análises que apresentaram $\mathrm{p} \leq 0,05$ e como tendência quando $0,05 \geq \mathrm{P} \leq 0,10$.

Quadro 1. Análise de comportamento: descrição das posturas e comportamentos adotados pelos bezerros antes e após o procedimento cirúrgico durante o experimento, baseada no modelo proposto por Thüer et al. (2007) e Marti et al. (2010)

\begin{tabular}{|c|c|}
\hline Comportamento & Descrição \\
\hline Pisoteio e escoiceamento & $\begin{array}{l}\text { Número de vezes em que os membros pélvicos são levantados e retornados ao solo com força ou batidos contra o } \\
\text { abdome com o animal em pé ou deitado. }\end{array}$ \\
\hline Lambedura & Número de vezes que o animal lambe a ferida cirúrgica com um dos membros traseiros levantados. \\
\hline \multirow[t]{2}{*}{ Inquietação } & Número de vezes que o animal deita e levanta sem permanecer numa posição por um pequeno período. \\
\hline & Posturas \\
\hline Postura normal & Levantar, andar ou movimentar sem anormalidades óbvias. \\
\hline Postura anormal & $\begin{array}{l}\text { Levantar ou andar com tremor, levantar com o dorso arqueado ou com os membros pélvicos levemente abertos ou } \\
\text { mais tracionados para trás, ou permanecer de joelhos. }\end{array}$ \\
\hline Decúbito normal & Decúbito esternal, com todos os membros embaixo do corpo, e a cabeça elevada ou apoiada. \\
\hline \multirow[t]{2}{*}{ Decúbito anormal } & Decúbito esternal ou lateral com extensão total ou parcial dos membros traseiros e cabeça elevada ou apoiada. \\
\hline & Índices \\
\hline Comportamento & $\begin{array}{l}\text { Soma de quantas vezes os animais manifestaram pisoteio, escoiceamento, lambedura e inquietação durante o } \\
\text { período observado. }\end{array}$ \\
\hline Postura & Porcentagem de posturas anormais em relação a todas as posturas adotadas durante o período observado. \\
\hline Escore Total & Somatória das frequências do comportamento e das porcentagens das posturas/ decúbito anormais. \\
\hline Sem dor-0 & $\begin{array}{ll}+2.20 & 0\end{array}$ \\
\hline Dor leve-1 & 1 a 20 \\
\hline Dor moderada-2 & 30 a 50 \\
\hline Dor severa-3 & 60 a 80 \\
\hline Dor intensa-4 & 90 а 120 \\
\hline
\end{tabular}


Quadro 2. Escala composta análogo-visual (EA): descrição de variações fisiológicas, comportamentais e expressões faciais adotadas por bezerros antes e após o procedimento cirúrgico. São Paulo, 2013

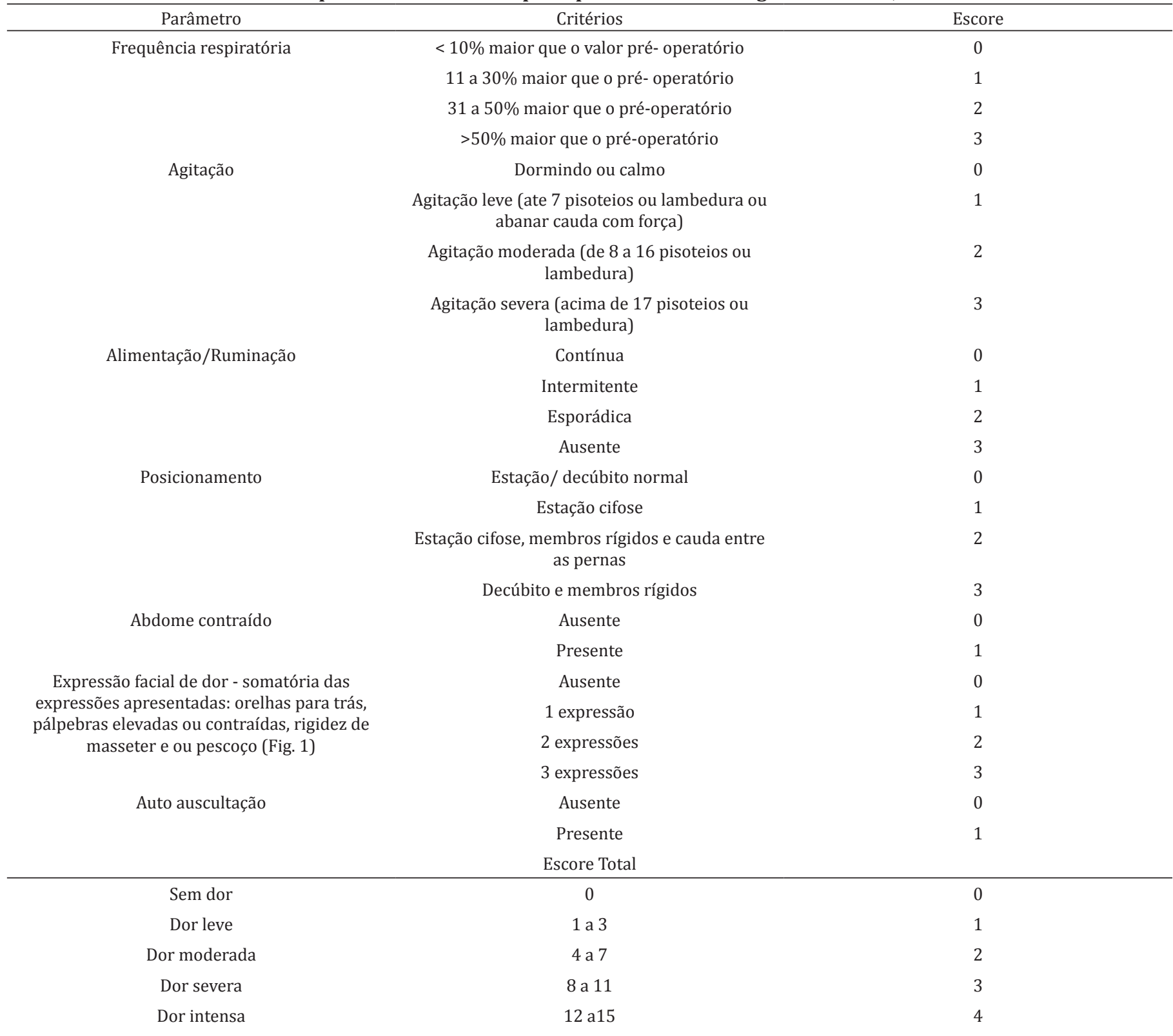

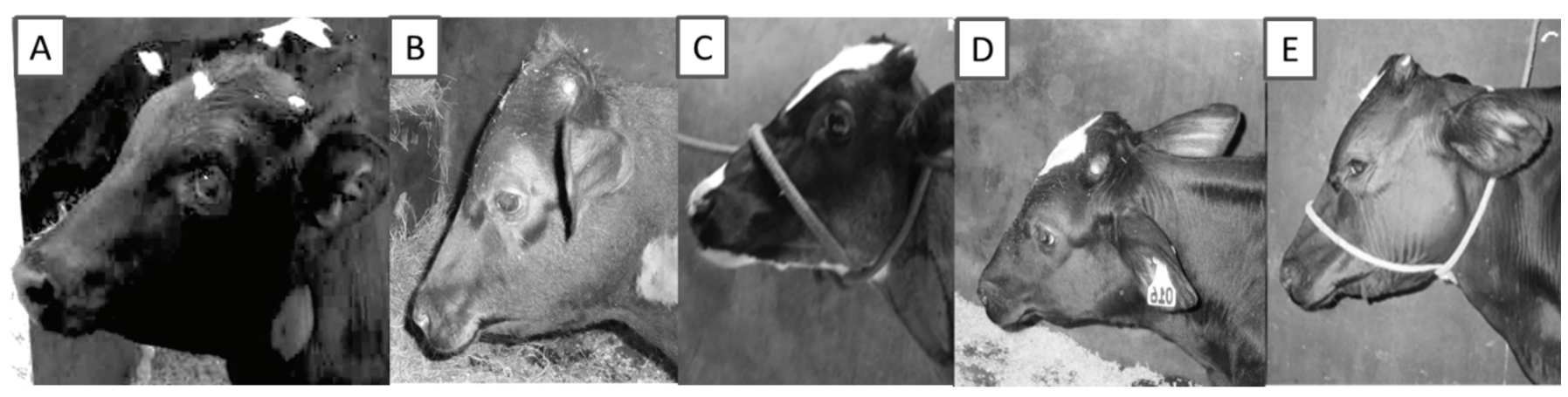

Fig.1. Expressão facial de dor. (A) Escore 0, ausência de dor. (B) Escore 1= pálpebras elevadas ou contraídas. (C) Escore 2 = orelha para trás e pálpebras elevadas ou contraídas. (D) Escore 2 = orelha para trás e rigidez de pescoço e masseter. (E) Escore $3=$ orelha para trás, pálpebras elevadas ou contraídas e rigidez de pescoço e masseter. 


\section{RESULTADOS}

Durante o estudo nenhum animal necessitou de medicamentos analgésicos, nem apresentou complicações como hemorragias profusas, formação de abscessos ou edema pronunciado da região. Desta maneira nenhum animal foi removido do estudo.

Os dados referentes às análises fisiológicas, comportamentais (Comp), expressão facial (Face) e escala análogo visual (EA) estão dispostos no Quadro 3.

Pode-se observar que o cortisol sérico apresentou um aumento biológico constante até 12 horas e depois tendeu a estabilidade, encontrando-se diferença apenas entre -30 e 12 horas $(\mathrm{P}=0,02)$. Em relação à frequência respiratória, notou-se que um aumento constante até 3 horas e depois declínio, com tendência a diferença entre 3 horas com os momentos $-30,72$ e 420 horas $(\mathrm{P}=0,06)$.

Em relação às análises de Comp, Face e EA, houve acordo acima de $90 \%$ entre os observadores. Para as avaliações de Comp, notou-se que alguns comportamentos relacionados à dor como pisoteio e lambedura foram manifestados em vários momentos em 50\% dos animais (4/8), apenas em um momento em $25 \%$ dos animas (2/8) e em nenhum momento em 25\% dos animais (2/8). Não foi observado abaixamento de cabeça em nenhum animal em nenhum dos momentos analisados e alguns animais agitaram a cauda constantemente (25\%-2/8), enquanto outros (76\%-6/8) não apresentaram este comportamento em nenhum momento. Pode-se observar que os animais manifestaram dor entre os momentos 1 a 24 horas, sendo o de maior intensidade às 6 horas, o qual diferenciou estatisticamente dos momentos -30, 24, 72 e 420 horas $(\mathrm{P}=0,0001)$, quando aplicada a escala baseada no modelo proposto por Thüer et al. (2007) e Marti et al. (2010).

Para as análises de expressão facial, notou-se certa dificuldade em identificar as unidades de ação facial relacionadas à elevação/contração da face medial e lateral das pálpebras e dilatação de narinas, conforme exposto no Quadro 4. Tal identificação era mais facilmente observada em imagens de perfil, no entanto a coloração do pelo escuro dificultou a visualização com detalhe destas áreas. Nenhum animal apresentou abertura de boca nos momentos avaliados. De maneira semelhante à Comp, pode-se observar que os animais manifestaram dor entre os momentos 1 a 24 horas, sendo o de maior intensidade às 6 horas, o qual diferenciou estatisticamente dos momentos $-30,72$ e 420 horas $(\mathrm{P}=0,002)$, quando aplicada no modelo proposto por Müller (2015).

Em relação à escala análogo visual (EA) verificou-se que apenas $12,5 \%$ dos animais (1/8) apresentaram em algum momento posição de auto auscultação, 87,5\%dos animais (7/8) apresentaram em algum momento abdômen contraído e $75 \%$ dos animais $(6 / 8)$ apresentaram em algum momento expressão facial de dor. Os demais parâmetros foram observados pelo menos em momento por todos os animais (8/8). A dor

Quadro 3. Identificação de dor baseado em análises fisiológicas, comportamentais, de expressão facial e análogovisual em bovinos antes e após a orquiectomia com protocolo analgésico

\begin{tabular}{|c|c|c|c|c|c|c|c|c|c|c|}
\hline \multirow{2}{*}{ Parâmetros } & & \multicolumn{8}{|c|}{ Tempo (horas) } & \multirow{2}{*}{$\mathrm{P}$} \\
\hline & & $-0,5$ & 1 & 3 & 6 & 12 & 24 & 72 & 420 & \\
\hline \multirow[t]{2}{*}{ Cortisol Sérico ng/ml } & Mediana & $3,44^{\mathrm{a}}$ & $5,98^{\mathrm{ab}}$ & $5,12^{\mathrm{ab}}$ & $5,66^{a b}$ & $8,78^{b}$ & 7,99 ab & $5,84^{a b}$ & $6,54^{a b}$ & 0,02 \\
\hline & IC & 2,3 & 2,5 & 1,5 & 3,3 & 5,6 & 3,9 & 3,0 & 3,2 & \\
\hline \multirow[t]{2}{*}{ FR } & Média & 26,5 & 31,4 & 32,0 & 30,3 & 30,3 & 28,6 & 26,0 & 24,8 & 0,06 \\
\hline & SEM & 0,95 & 4,86 & 2,23 & 3,85 & 1,20 & 2,53 & 1,40 & 1,30 & \\
\hline Escore Comp & & 0,00 & 2,84 & 2,36 & 3,92 & 2,72 & 1,68 & 1,26 & 0,00 & \\
\hline \multirow[t]{3}{*}{ Escore EA } & Mediana & $0^{\mathrm{a}}$ & $2^{\mathrm{ab}}$ & $3^{\mathrm{b}}$ & $3^{\mathrm{b}}$ & $2^{\mathrm{ab}}$ & $2^{\mathrm{ab}}$ & $1^{\mathrm{a}}$ & $1^{\mathrm{a}}$ & 0,0001 \\
\hline & IC & 0,00 & 0,70 & 2,10 & 1,41 & 1,12 & 0,85 & 0,84 & 0,01 & \\
\hline & & 0,00 & 4,30 & 4,76 & 4,53 & 3,94 & 4,28 & 2,93 & 2,02 & \\
\hline Escore Face & Mediana & $0^{\mathrm{a}}$ & $1,37^{\mathrm{ab}}$ & $1,37^{\mathrm{ab}}$ & $2,12^{\mathrm{b}}$ & $1,62^{\mathrm{ab}}$ & $0,62^{\mathrm{ab}}$ & $0^{\mathrm{a}}$ & $0^{\mathrm{a}}$ & 0,002 \\
\hline
\end{tabular}

$\overline{\mathrm{IC}}=$ Intervalo de confiança, FR $=$ frequência respiratória, Comp = Comportamento, EA = escala análogo visual, Face = expressão facial. Letras diferentes na mesma linha indicam diferença estatística.

Quadro 4. Imagens não hábeis para avaliação para cada unidade de ação facial

\begin{tabular}{|c|c|}
\hline Unidade de ação facial & Sem visualização para classificação de \% (n. i imagens (sem avaliação/n. total de imagens) \\
\hline Colocação das orelhas para trás & $0 \%(0 / 64)$ \\
\hline Dilatação das narinas & $24,43 \%(15 / 64)$ \\
\hline E/C face medial das pálpebras & $21,87 \%(14 / 64)$ \\
\hline E/C da face lateral das pálpebras & $21,87 \%(14 / 64)$ \\
\hline Abertura da boca & $4,68 \%(3 / 64)$ \\
\hline
\end{tabular}


foi identificada entre os momentos 1 até 72 horas, estando com maior intensidade as 3 e 6 horas após o procedimento, os quais diferiram estatisticamente de momento basal $(-30)$ e de 420 horas $(\mathrm{P}=0,0001)$.

Em relação à correlação entre os diferentes modelos de identificação de dor em bovinos submetidos à orquiectomia cirúrgica, pode-se observar que o cortisol apresentou correlação positiva fraca com frequência respiratória $(\mathrm{r}=0,3 \mathrm{P}=0,01)$, com Comp $(r=0,25, P=0,04)$ com Face $(r=0,22, P=0,07)$ e com EA (correlação, $r=0,15, P=0,23$ ). Entre as escalas, observou-se correlação positiva maior entre a Comp e $\mathrm{EA}(\mathrm{r}=0,58, \mathrm{P}=0,0001)$, seguida por menores correlações entre a Face e EA ( $r=0,38, P=0,02)$ e Face e Comp $(r=0,31, P=0,09)$. As correlações entre as escalas e frequência respiratória (FR) também foram fracas (FR e Comp $\mathrm{r}=0,23 \mathrm{P}=0,05$; FR e Face $r=0,03, P=0,75$; FR e EA $r=0,38, P=0,001$ ).

\section{DISCUSSÃO}

Procedimentos de orquiectomia são dolorosos e ainda não existe um consenso sobre qual protocolo analgésico ou cirúrgico é ideal (Becker et al. 2012). Tal fato implicou em ausência de grupo controle, pois preconizou respeitar os preceitos de éticas e de bem-estar evitando-se grupo sem analgesia. Além disso, o foco do trabalho não foi comparar diferentes técnicas cirúrgicas ou analgésicas, optando-se desta maneira utilizar o animal como controle dele mesmo, realizando-se um estudo longitudinal, no qual o momento basal (M-30) foi o momento de comparação entre os demais momentos.

Para as análises do cortisol sérico, encontra-se ampla variação de resultados nos trabalhos que utilizaram a mesma metodologia cirúrgica e analgésica que a presente pesquisa. Observou-se aumento do cortisol sérico desde a primeira meia hora pós cirúrgica (Ballou et al. 2013), entre 4 a 5 horas (Onda et al. 2012), às 72 horas (Ballou et al. 2013) ou não encontrou-se aumento após o procedimento (Stafford et al. 2002), sendo todos estes resultados diferentes da presente pesquisa, cujo aumento foi 12 horas após o procedimento.

0 pico de cortisol sérico indica uma resposta nociceptiva, mediada por uma série de hormônios que ativam mecanismos compensatórios causando taquicardia e taquipneia (Molony et al. 1995, Fitzpatrick et al. 2006). Desta maneira era esperado que o aumento da frequência respiratória ocorresse cronologicamente ao aumento do cortisol sérico. Possivelmente isto não ocorreu uma vez que o cortisol pode ser influenciado pela simples manipulação do animal, como a coleta de sangue (Becker et al. 2012) e a magnitude de sua resposta é influenciada pelo temperamento do animal, o que aumenta a variabilidade de respostas entre cada individuo (Hulbert et al. 2011). A frequência respiratória foi aferida por inspeção, portanto sem a manipulação do animal, o que sugere que esta variável sofreu menos influência externa que o cortisol sérico (Becker et al. 2012).

Tal qual a frequência respiratória, a aplicação das escalas também foi feita sem a manipulação do animal. 0 fato de serem capturadas por filmagem, ainda permite o uso de recursos como pausar, retornar ou amentar o tamanho das imagens, garantindo maior confiabilidade e transparência aos critérios adotados, bem como identificar novos comportamentos dos que os inicialmente propostos (Millman 2013, Dalla Costa et al. 2014).

A escala de comportamento foi baseada em relatos da literatura que indicaram que a dor visceral e a dor somática causadas pela orquiectomia possam ser identificadas e graduadas por lambedura do local afetado, escoiceamento, pisoteio, agitação da cauda, abaixamento da cabeça e posturas antiálgicas (Thüer et al. 2007, Marti et al. 2010). Apesar de estes comportamentos serem classificados como uma medida robusta de identificação dolorosa (Millman 2013) observou-se que os bezerros demonstravam também outros comportamentos como taquipneia, graduações diferentes de posicionamento antiálgicos incluindo abdome tenso e posição de auto auscultação além de expressões faciais de dor, permitindo que se elaborasse uma escala análogo visual mais abrangente (Quadro 2).

Cabe ressaltar que alguns animais da presente pesquisa não demostraram em nenhum momento pisoteio, lambedura do local afetado, agitação da cauda ou abaixamento da cabeça. Estes resultados também foram descritos por Marti et al. (2010), que concluíram que os animais castrados como mesmo protocolo analgésico não sentiram dor pós cirúrgica.

Discordando de Marti et al. (2010) e Millman (2013), acredita-se que a escala de comportamento não foi tão robusta para identificar dor e por isso encontrou-se certa discrepância na identificação de maior graduação de dor entre as escalas de comportamento e a análogo visual.

Tanto a escala de comportamento como a escala de expressão facial identificaram maior graduação de dor 6 horas após o procedimento. E apesar das análises de expressão facial demostrarem ser uma boa forma de caracterizar e graduar dor em bovinos (Millman 2013, Müller 2015), a metodologia proposta não permitiu que as faces fossem analisadas com detalhes em todos os animais nos momentos propostos.

Tal metodologia foi sugerida por Dalla Costa et al. (2014), na qual fotos eram removidas dos vídeos, o que diminui-se o tempo dispendido para estas análises, já que as escalas de Comp e EA foram realizadas em vídeos de 10 minutos para cada animal, repetidos por 8 momentos. Assim o tempo dispendido para as análises de dor foi, portanto, de no mínimo 800 minutos, e para análise da expressão facial foi de aproximadamente 40 minutos.

Além disso, quando as fotos são capturadas imediatamente da face do animal, como a metodologia proposta por Müller (2015), existe interação do examinador com o animal, o que pode interferir no comportamento ou a expressão facial dos mesmos. Esta interferência do avaliador na expressão facial foi sugerida por Leach et al. (2012), ao explicar que animais classificados como presa tem a habilidade de mascarar sinais óbvios de dor para escapar do predador.

Além disso, ambas as metodologias relatam que a coloração do pelo do animal interferiu na qualidade da imagem dificultando a análise das expressões faciais (Dalla Costa et al. 2014, Müller 2015), tal qual no presente trabalho.

Notou-se também que os animais não vocalizaram nos momentos pós-cirúrgicos, o que não pontuou escore de abertura da boca no presente estudo. Diferente dos achados de Müller (2015), que considerou esta unidade de ação facial (UAF) importante em seu estudo. Tal diferença se deve ao fato de que no trabalho de Müller (2015), as observações eram feitas imediatamente no momento da aplicação do estimulo doloroso. Desta maneira considerou-se que esta UAF não se aplica ao presente estudo. Apesar das vantagens relatadas em relação à metodologia proposta para caracterizar Face, verificamos que as fotos não foram capazes de substituir os 
vídeos, não apenas devido à perda de qualidade da imagem, mas também porque houve momentos nos vídeos em que as expressões faciais eram mais evidentes. Ainda notou-se a elevação/contração das faces medias e laterais das pálpebras nem sempre eram bem observadas e foi o principal ponto de discordância entre os observadores. Já a rigidez de pescoço e/ou de masseter era bem observada nos vídeos e são UAF importante para avaliação de dor em equinos (Dalla Costa et al. 2014).

Desta maneira, acredita-se que limitar a análise de graduação de dor a detalhes de face, ou de determinados tipos de comportamentos como pisoteio ou lambedura podem minimizar a identificação da dor do animal, seja por perda de qualidade da metodologia proposta, pelo temperamento do animal que mascaram alguns comportamentos ou por interferência do avaliador na expressão facial ou em seu comportamento.

Por isso a identificação de dor baseada em interpretação de múltiplas respostas, como os itens elencados para montar a escala análogo visual, aumenta a acurácia e a reprodutibilidade do teste (Thüer et al. 2007, Müller 2015).

Como a EA foi montada em comportamentos e expressões faciais identificadas nos vídeos, notou-se maiores índices de correlação entre estas três análises e maior concordância entre a identificação de maior graduação de dor entre estas três metodologias. Até o momento esta é a primeira vez eu uma escala análogo visual é montada para bovinos, e não se sabe se ela pode ser utilizada para outros ruminantes ou para avaliação de dor de outros protocolos cirúrgicos.

\section{CONCLUSÕES}

Conclui-se que todos os modelos propostos foram capazes de identificar dor pós-orquiectomia em bovinos; no entanto a graduação de maior dor foram em momentos pós-cirúrgicos distintos conforme o parâmetro avaliado.

Houve fraca correlação entre os modelos propostos, pois se encontrou limitações para a maioria das metodologias avaliadas, concluindo-se que a associação entre os vários parâmetros de dor, tal qual o utilizado na escala análogo visual, aumenta a acurácia em identificar a dor após orquiectomia em bovinos.

Agradecimentos.- Apoio financeiro FAPESP, projeto 2011/21632-0.

\section{REFERÊNCIAS}

Anderson N. 2007. Castration of calves. Order no. 07-029, Factsheet Animal Science, Ministry of Agriculture Food and Rural Affairs, Ontario, p.420-426.

Ballou M.A., Sutherland M.A., Brooks T.A., Hulbert L.E., Davis B.L. \& Cobb C.J. 2013. Administration of anesthetic and analgesic prevents the suppression of many leukocyte responses following surgical castration and physical dehorning. Vet. Immunol. Immunopathol.151(3/4):285-293. http://dx.doi. org/10.1016/j.vetimm.2012.11.018. PMid:23270586.

Becker J., Doherr M.G., Bruckmaier R.M., Bodmer M., Zanolari P. \& Steiner A. 2012. Acute and chronic pain calves after different methods of rubberring castration. Vet. J. 194(3):380-385. http://dx.doi.org/10.1016/j. tvjl.2012.04.022. PMid:22647596.

Bellinazzi J.B., Bertagnon H.G., Batista C.F., Santos B.P., Lima M.G.B., Lima D.M., Benesi F.J. \& Della Libera A.M.M. 2013. Efeitos do estresse da orquiectomia na citologia broncoalveolar de bezerros da raça Holandesa. Pesq. Vet. Bras. 33(Supl.1):93-98. http://dx.doi.org/10.1590/S0100-736X2013001300015.
Bloom T. \& Friedman H. 2013. Classifying dogs' (Canis familiaris) facial expressions from hotographs. Behav. Processes 96:1-10. http://dx.doi. org/10.1016/j.beproc.2013.02.010. PMid:23485925.

Chambers C.T., Finley G.A., McGrath P.J. \& Walsh T.M. 2003. The parents' postoperative pain measure: replication and extension to 2-6-year-old children. Pain 105(3):437-443. http://dx.doi.org/10.1016/S03043959(03)00256-2. PMid:14527704.

Costa M.J.R. \& Cromberg V.U. 1997. Alguns aspectos a serem considerados para melhorar o bem-estar de animais em sistema de pastejo rotacionado, p.273-296. In: Peixoto A.M., Moura J.C. \& Faria V.C. (Eds), Fundamentos do Pastejo Rotacionado. FEALQ Piracicaba.

Dalla Costa E., Minero M., Lebelt D., Stucke D., Canali E. \& Leach M.C. 2014. Development of the Horse Grimace Scale (HGS) as a pain assessment tool in horses undergoing routine castration. PLoS One 9(3):e92281. http:// dx.doi.org/10.1371/journal.pone.0092281. PMid:24647606.

Ekman P., Freisen W.V. \& Ancoli S. 1980. Facial signs of emotional experience. J. Pers. Soc. Psychol. 39(6):1125-1134. http://dx.doi.org/10.1037/h0077722.

Fantoni D.T. \& Mastrocinque S. 2002. Fisiopatologia da dor, p.323-334. In: Fantoni D.T. \& Cortopassi S.R.G. (Eds), Anestesia em Cães e Gatos. Roca, São Paulo.

Fitzpatrick J., Scott M. \& Nolan A. 2006. Assessment of pain and welfare in sheep. Small Rumin. Res. 62(1/2):55-61. http://dx.doi.org/10.1016/j. smallrumres.2005.07.028.

Hulbert L.E., Carroll J.A., Burdick N.C., Randel R.D., Brown M.S. \& Ballou M.A. 2011. Innate immune responses of temperamental and calm cattle after transportation. Vet. Immunol. Immunopathol. 143(1/2):66-74. http:// dx.doi.org/10.1016/j.vetimm.2011.06.025. PMid:21726904.

Lay Jr D.C., Friend T.H., Grissom K.K., Bowers C.L. \& Mal M.E. 1992. Effects of freeze or hot-iron branding of angus calves on some physiological and behavioral indicators of stress. Appl. Anim. Behav. Sci. 33(2/3):137-147. http://dx.doi.org/10.1016/S0168-1591(05)80003-6.

Leach M.C., Klaus K., Miller A.L., Scotto di Perrotolo M., Sotocinal S.G. \& Flecknell P.A. 2012. The assessment of post-vasectomy pain in mice using behaviour and the mouse grimace scale. PLoS One 7(4):e35656. http:// dx.doi.org/10.1371/journal.pone.0035656. PMid:22558191.

Marti S., Velarde A., De la Torre J.L., Bach A., Aris A., Serrano A., Manteca X. \& Devant M. 2010. Effects of ring castration with local anesthesia and analgesia in Holstein calves at 3 months of age on welfare indicators. J. Anim. Sci. 88(8):2789-2796. http://dx.doi.org/10.2527/jas.2009-2408. PMid:20418456.

Millman S.T. 2013. Behavioral responses of cattle to pain and implications for diagnosis, management, and animal welfare. Vet. Clin. N. Am., Food Anim. Pract. 29(1):47-58. http://dx.doi.org/10.1016/j.cvfa.2012.11.007.

Molony V., Kent J.E. \& Robertson I.S. 1995. Assessment of acute and chronic pain after different methods of castration of calves. Appl. Anim. Behav. Sci. 46(1/2):33-48. http://dx.doi.org/10.1016/0168-1591(95)00635-4.

Müller B.R. 2015. Dor em bovinos na marcação a ferro quente: expressão facial, outros indicadores comportamentais e fisiológicos e a percepção dos produtores. Dissertação de Mestrado em Ciências Veterinárias, Universidade Federal do Paraná, Curitiba. 77p.

Onda K., Emura Y., Saito A., Ikeyama T., Sato R., Ochiai H., Kaneko K., Iriki T. \& Wada Y. 2012. Sodium salicilate attenuates acute stresse response to castration in calves. J. Anim. Vet. Adv. 11(10):1574-1577. http://dx.doi. org/10.3923/javaa.2012.1574.1577.

Robertson I.S., Kent J.E. \& Molony V. 1994. Effect of different methods of castration on behaviour and plasma cortisol in calves of three ages. Res. Vet. Sci. 56(1):8-17. http://dx.doi.org/10.1016/0034-5288(94)90189-9. PMid:8146458. 
Stafford K.J., Mellor D.J., Todd S.E., Bruce R.A. \& Ward R.N. 2002. Effects of local anaesthesia or local anaesthesia plus a non-steroidal anti-inflammatory drug on the acute cortisol response of calves to five different methods of castration. Res. Vet. Sci. 73(1):61-70. http://dx.doi.org/10.1016/S00345288(02)00045-0. PMid:12208108.

Tate A.J., Fischer H., Leigh A.E. \& Kendrick K.M. 2006. Behavioural and neurophysiological evidence for face identity and face emotion processing in animals. Philos. Trans. R. Soc. Lond., B, Biol. Sci. 361(1476):2155-2172. http://dx.doi.org/10.1098/rstb.2006.1937. PMid:17118930.
Thüer S., Mellema S., Doherr M.G., Wechsler B., Nuss K. \& Steiner A. 2007. Effect of local anaesthesia on short- and long-term pain induced by two bloodless castration methods in calves. Vet. J. 173(2):333-342. http:// dx.doi.org/10.1016/j.tvjl.2005.08.031. PMid:16223591.

Ting S.T.L., Earley B. \& Crowe M.A. 2003. Effect of repeated ketoprofen administration during surgical castration of bulls on cortisol, immunological function, feed intake, growth, and behavior. J. Anim. Sci. 81(5):1253-1264. http://dx.doi.org/10.2527/2003.8151253x. PMid:12772853. 